\title{
Synthesis of Polycrystalline Cristobalite Balls through Reversible Replication of Colloid-Imprinted Carbon
}

\author{
Chy Hyung Kim* and Teresa $\mathrm{Oh}^{\dagger}$ \\ Department of Chemistry, Cheongju University, Cheongju 360-764, Korea. E-mail: chkim@cju.ac.kr \\ †Department of Electronics and Information Engineering, Cheongju University, Cheongju 360-764, Korea \\ Received March 3, 2011, Accepted July 18, 2011
}

Key Words : Colloid-imprinted carbon template, Cristobalite ball, Reversible replication method, Sodium silicate solution

Mesoporous carbon structures have been recognized these days due to their potential applications in catalysis, adsorption, energy storage, and as other advanced materials. ${ }^{1-7}$ Since a class of mesoporous carbon has been formed through the nanocasting replication of mesoporous silica, ${ }^{8-11}$ the carbon replica has been successfully employed as a further template to produce inverse replica of mesoporous silica. ${ }^{12}$ Recently, mesoporous carbons with uniform pore size distributions have been formed through the imprinting of pitch or the use of poly(vinylidene chloride-co-vinyl chloride) with colloidal silica. ${ }^{13,14}$ Since the article was published, ${ }^{13}$ the zeolite (ZSM-5) with the uniform crystal size has been synthesized at $180{ }^{\circ} \mathrm{C}$ and calcined at $570{ }^{\circ} \mathrm{C}$, successively, by using the colloid-imprinted carbon (CIC) as a template. ${ }^{15}$ However, the carbon replica has not been utilized as a template at a very high temperature to obtain an inverse replica.

In this study, we present the synthesis of crystalline form of silicate balls with uniform size from an amorphous precursor by the reversible replication method through the CIC template at a very high temperature of $1200{ }^{\circ} \mathrm{C}$, but lower than the usual crystallization temperature, 1470-1727 ${ }^{\circ} \mathrm{C}$. ${ }^{16}$ The precursor, amorphous sodium silicate solution, was converted to a stable crystalline form of silica, cristobalite, through the CIC template. Cristobalite undergoes a passivation process and enhances the thermal stability in the formation of improved crucibles which are used in crystal growth. Cristobalite is used as a mechanically-cleaning abrasive component in liquid-cleaning compositions. It possesses characteristics of a higher degree of hardness and a lower specific gravity than conventional mineral powders used as abrasive substances. ${ }^{17}$ It can be used not only as a filler in paints and plastics, but also as an investment for dental ceramics, ${ }^{18}$ as a component of heat resistant caulking compositions, as catalyst carriers, as extracting agents, and as a component of refractories. ${ }^{19}$ The cristobalite is crystallized at $1470-1727{ }^{\circ} \mathrm{C}$, respectively, and it continues to exist at room temperature..$^{16,19}$

Colloid-imprinted carbon (CIC) was prepared through the imprinting of pitch with colloidal silica according to the literature. $^{13,15}$ Figure 1 illustrates that the imprinting of colloidal silica creates pores in the mesophase pitch particle.
Figure 1 also shows TEM image of CIC template prepared through the mesophase pitch and Snowtes-ZL colloidal silica, which matches well with the illustration of the synthesis of CIC in the figure. The pore size is also in good agreement with the average pore size of colloidal silica, Snowtes-ZL $(85 \mathrm{~nm})$. As shown in the figure, the wall between the pores is thin and the thin wall will be ruptured when a certain stimulus is exerted on the wall. The upper diagram in Figure 2 is a scheme for the synthesis of crystalline cristobalite balls by using the CIC template. According to the measurement of nitrogen adsorptiondesorption isotherms, the pore volume of CIC is $0.65 \mathrm{~cm}^{3} / \mathrm{g}$. The density of sodium silicate solution $(\sim 27 \mathrm{wt} \%$ silica $)$ is $1.39 \mathrm{~g} / \mathrm{cm}^{3}$. Thus, the added weight of sodium silicate corresponds to $75 \%$ of the pore volume of CIC to prevent the formation of silicate bulk. Figure 2 also shows a real TEM image of cristobalite balls synthesized from the amorphous sodium silicate solution by following the process of the scheme in the figure. The TEM image of cristobalite balls in Figure 2 is different from the result of the scheme to a certain extent in that the balls are associated with each other.
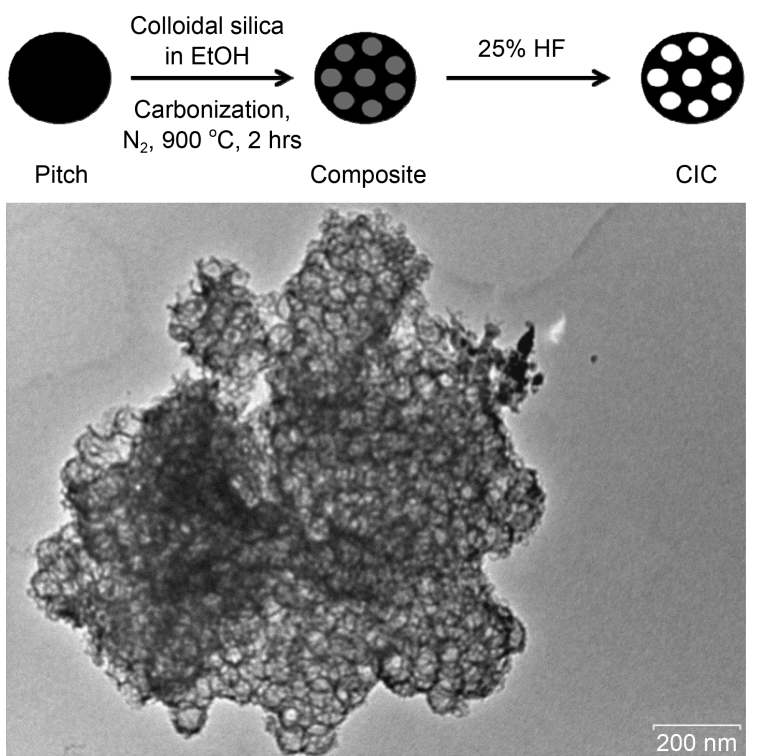

Figure 1. Scheme for the synthesis of CIC, and TEM image of CIC with a scale size of $200 \mathrm{~nm}$. 


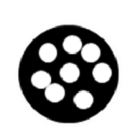

$\mathrm{CIC}$

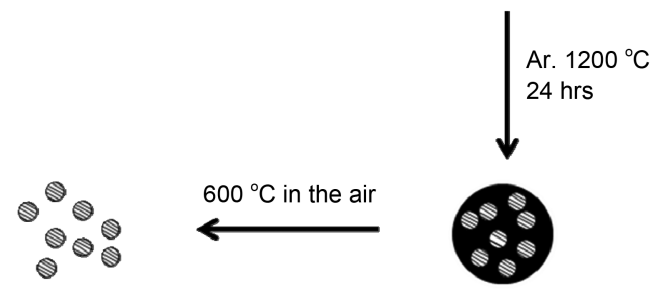

cristobalite balls

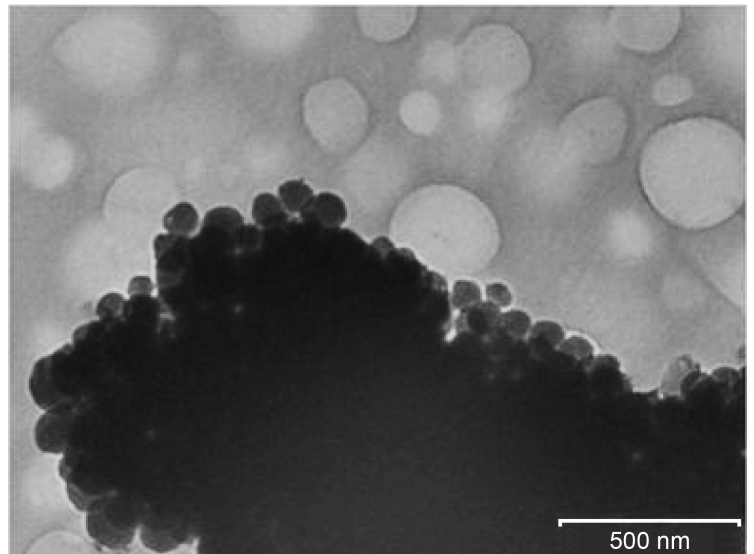

Figure 2. Scheme for the synthesis of polycrystalline cristobalite balls by using the CIC template, and TEM image of cristobalite balls with a scale size of $500 \mathrm{~nm}$ which are synthesized from the amorphous sodium silicate solution.

The balls stick together because the balls occupied in pores of CIC can be diffused into the neighboring pores at high temperature through the thin wall. However, some balls of uniform size are still found on the surface part in the TEM image, indicating that the CIC template contributes to maintaining the ball shape by inhibiting the attachment of the balls to some degree during the crystallization of the amorphous precursor. When the wall between the pores of the CIC is formed thick enough to prevent the diffusion of the balls at the high temperature, uniform meso-sized balls will be produced without sticking to each other.

Although the stable cristobalite has been known to be synthesized at above $1470{ }^{\circ} \mathrm{C},{ }^{16,19}$ cristobalite balls are mainly synthesized at $1200{ }^{\circ} \mathrm{C}$ in this study because the meso-sized balls occupied in meso-sized pores of CIC show enhanced reactivities during crystallization based on their larger surface area-to-volume ratio. Thus, the crystallization of the balls occurs at a lower temperature than in the case of bulk form. This can be proved by comparing the XRD patterns of the ball and the bulk type. Therefore, another silicate of bulk form was prepared without using the CIC template by following the same procedure. Figure 3(a) and 3(b) reveal the XRD patterns of the synthesized ball through CIC and bulk prepared without using CIC. The XRD pattern of cristobalite from JCPDS Index is shown in Figure 3(c) as

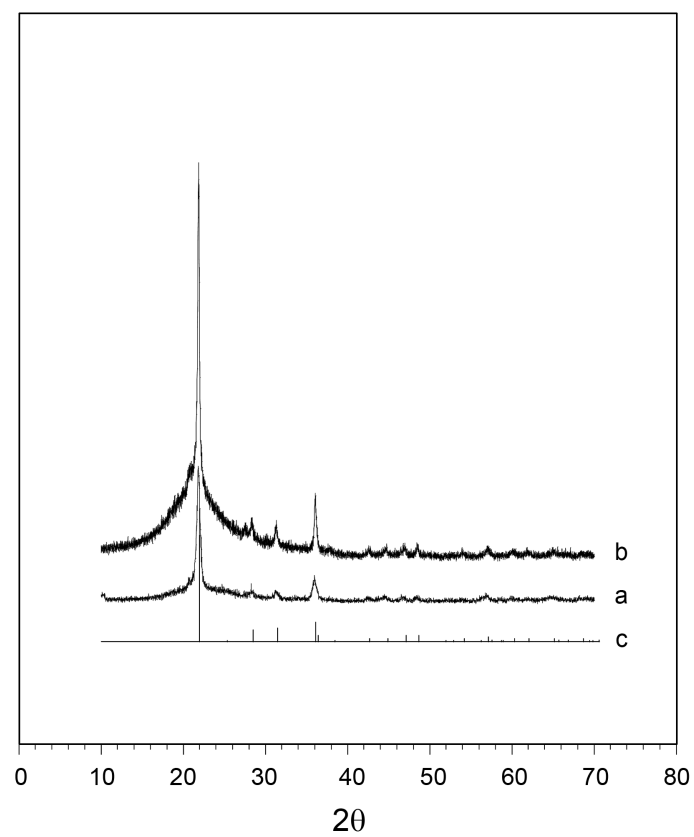

Figure 3. XRD patterns of (a) the ball synthesized using the CIC template; (b) the bulk fabricated without using the CIC template; and (c) cristobalite from JCPDS Index.

a reference, where the XRD pattern of the synthesized balls (Figure 3(a)) matches well with the pattern of the cristobalite index. In other words, the cristobalite structure is observed both in the XRD patterns of the ball form and the bulk form, indicating that the amorphous sodium silicate solution is an available precursor for the synthesis of cristobalite when the precursor is treated at $\mathrm{pH}$ of $\sim 6.5$ and calcined at $1200{ }^{\circ} \mathrm{C}$. However, the bulk form prepared without using CIC demonstrates a more amorphous XRD pattern than that of the ball form and the component of tridymite is included as an impurity in the pattern. ${ }^{16}$ Thus, when the CIC template is used, a higher content of cristobalite can be observed in meso-sized balls.

The specific BET surface area of $62 \mathrm{~m}^{2} / \mathrm{g}$ and the pore volume of $0.65 \mathrm{~cm}^{3} / \mathrm{g}$ for the CIC template were obtained from nitrogen adsorption-desorption isotherms. On the other hand, the textural properties of cristobalite balls were different from those of CIC and showed a very low value of pore volume, $0.04 \mathrm{~cm}^{3} / \mathrm{g}$, because the cristobalite balls became dense at $1200{ }^{\circ} \mathrm{C}$ and mesoporous CIC was already burned out in the air. In particular, the association of cristobalite balls through the diffusion of the balls at a high temperature caused the level of surface area to be low, with a specific BET surface area of $9.6 \mathrm{~m}^{2} / \mathrm{g}$.

In summary, the imprinting of mesophase pitch with colloidal silica provided a simple ways in synthesizing uniform and adjustable carbon pore size and preparing applicable ceramic, metallic, or multi-component balls through the reversible replication of colloid-imprinted carbon. By using the CIC template, most of the cristobalite-structured balls synthesized from the amorphous precursor (sodium silicate solution) could be observed at $1200^{\circ} \mathrm{C}$ which is lower than the conventional crystallization temperature. 
Although the balls occupying the pores diffuse into the next pores and associate together through the thin wall between the pores at $1200{ }^{\circ} \mathrm{C}$, the CIC template still contributes to maintaining the ball shape to some extent by inhibiting the attachment of the balls. When the thickness of the wall and the synthetic temperature are adjusted, uniform meso-sized balls of various compounds will be produced without sticking together. Thus, the reversible replication method will be very useful for the future electrochemical applications, catalysis, and for synthesizing various functional meso-sized ball materials.

\section{Experimental Section}

Colloid-imprinted Carbon (CIC). Mesophase pitch (Mitsubishi Chemicals) as a carbon source and Snowtes-ZL (average particle diameter of $85 \mathrm{~nm}$ ) as a colloidal silica were obtained. Silica-to-pitch weight ratio of 1.6 was selected to form the imprinted carbon replica effectively. $15.0 \mathrm{~g}$ of mesophase pitch was dispersed in $150 \mathrm{~g}$ of EtOH and $60.0 \mathrm{~g}$ of colloidal silica suspension (40 wt \% silica) was added, and then the mixture was stirred overnight at $50{ }^{\circ} \mathrm{C}$. The solvent evaporated at the same temperature during the stirring stage. The mixture was further dried at $270{ }^{\circ} \mathrm{C}$, a slightly higher temperature than the softening point of the pitch under nitrogen flow for 1 hour. Meanwhile, the colloidal particles penetrated into the pitch and formed a composite. The composite was carbonized at $900{ }^{\circ} \mathrm{C}$ for $2 \mathrm{hrs}$ under nitrogen flow, and then washed with $25 \%$ of $\mathrm{HF}$ to remove the silica.

Silicate Ball. For the synthesis of cristobalite ball through CIC as a template, $0.8 \mathrm{~g}$ of CIC was initially soaked in EtOH. Following the slow evaporation of EtOH from the impregnated CIC at room temperature, $2.0 \mathrm{~g}$ of sodium silicate solution dissolved in $1 \mathrm{~mL}$ of $\mathrm{H}_{2} \mathrm{O}$ was added to CIC and shaken on a warm plate until the mixture was dried, where the added weight of sodium silicate corresponds to $75 \%$ of the pore volume of CIC to prevent the formation of silicate bulk. After that, the mixture was buffered to $\mathrm{pH}$ of $\sim 6.5$ with $7.4 \mathrm{~mL}$ of $1.0 \mathrm{M}$ acetic acid, shaken at $60{ }^{\circ} \mathrm{C}$ for one day, filtered with water thoroughly, and dried. The resulting composite was heated under argon flow at $1200{ }^{\circ} \mathrm{C}$ for $24 \mathrm{hrs}$ for the crystallization of silica and in the air at $600{ }^{\circ} \mathrm{C}$ for $12 \mathrm{hrs}$ to remove the CIC template. Another silicate of bulk type was also prepared without using CIC template by the same procedure, and then x-ray was used to compare the XRD patterns of the both forms.

XRD patterns were obtained by utilizing a Rigaku Rotaflex diffractometer with $\mathrm{Cu} \mathrm{K} \alpha$ radiation. TEM studies were carried out on a JEOL 100 CX instrument. Samples for TEM studies were prepared by dipping a carbon-coated $\mathrm{Cu}$ grid into a suspension of samples in EtOH. For the textural property measurements of CIC and the cristobalite ball, the nitrogen adsorption-desorption isotherms were applied at $-196^{\circ} \mathrm{C}$ on a Micromeritics Tristar instrument.

\section{References}

1. Hyeon, T. H.; Han, S. J.; Sung, Y.-E.; Park, K.-W.; Kim, Y.-W. Angew. Chem. Int. Ed. 2003, 42, 4352.

2. Kyontani, T. Carbon 2000, 38, 269.

3. Han, S. J.; Yun, Y. K.; Park, K.-W.; Sung, Y.-E.; Hyeon, T. H. $A d v$. Mater. 2003, 15, 1922.

4. Fang, B.; Kim, J. H.; Yu, J.-S. Electrochem Commun. 2008, 10, 659.

5. Piao, Y.; Lee, D. H.; Kim, J. B.; Kim, J. Y.; Hyeon T. H.; Kim, H.S. Analyst 2009, 134, 926.

6. Yu, J. S.; Kang, S.; Yoon, S. B.; Chai, G. J. Am. Chem. Soc. 2002, $124,9382$.

7. Piao, Y.; Kim, H. S.; Sung, Y.-E.; Hyeon, T. H. Chem. Comm. 2010, 46, 118

8. Ryoo, R.; Joo, S. H.; Jun, S. J. Phys. Chem. B 1999, 103, 7743.

9. Ryoo, R.; Joo, S. H.; Kruk, M.; Jaroniec, M. Adv. Mater. 2001, 13, 677.

10. Kim, S. S.; Pinnavaia, T. J. Chem. Commun. 2001, 2418.

11. Lee, J. W.; Han, S. J.; Hyeon, T. H. J. Mater. Chem. 2004, 14, 478.

12. Kang, M.; Yi, S. H.; Lee, H. I.; Yie, J. E.; Kim, J. M. Chem. Commun. 2002, 1944.

13. Li, Z.; Jaroniec, M. J. Am. Chem. Soc. 2001, 123(37), 9208.

14. Choma, J.; Zawiœlak, A.; Górka, J. Adsorption 2009, $15(2), 167$.

15. Kim, S.-S.; Shah, J.; Pinnavaia, T. J. Chem. Mater. 2003, 15, 1664.

16. Shinohara, Y.; Kohyama, N. Industrial Health 2004, 42, 277.

17. Pastor et al. U.S. Patent, Patent No. 4429009 Jan. 31, 1984.

18. Taniichiro, Y.; Akiisa, M.; Takayuki, H.; Ken'ichi, K.; Yasushi, H.; Hiroshi, N. J. the Japanese Society for Dental Materials and Devices 2002, 21,126.

19. Kaduk, J. A. U.S. Patent, Patent No. 4395388 July 26, 1983. 